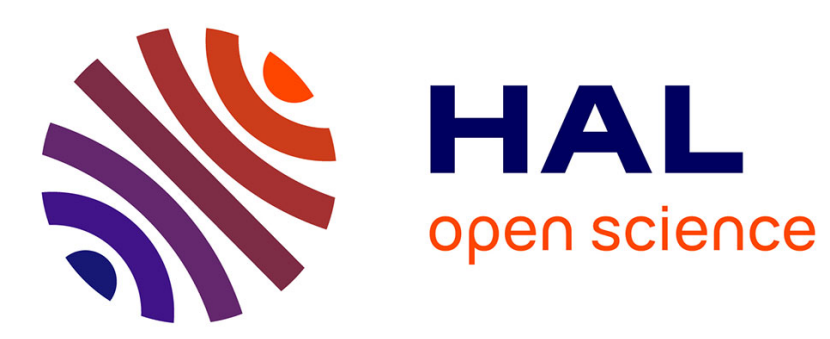

\title{
Emotion, collectif et lien social : vers une approche sociologique du don humanitaire
}

\author{
Catherine Dessinges
}

\section{To cite this version:}

Catherine Dessinges. Emotion, collectif et lien social: vers une approche sociologique du don humanitaire. Revue du MAUSS, 2008, 32, pp.203-221. hal-00590758

\section{HAL Id: hal-00590758 \\ https://hal.science/hal-00590758}

Submitted on 5 May 2011

HAL is a multi-disciplinary open access archive for the deposit and dissemination of scientific research documents, whether they are published or not. The documents may come from teaching and research institutions in France or abroad, or from public or private research centers.
L'archive ouverte pluridisciplinaire HAL, est destinée au dépôt et à la diffusion de documents scientifiques de niveau recherche, publiés ou non, émanant des établissements d'enseignement et de recherche français ou étrangers, des laboratoires publics ou privés. 


\section{Emotion, collectif et lien social : vers une approche sociologique du don humanitaire. \\ in L'amour des autres, Care, compassion et humanitarisme, Revue du Mauss $n^{\circ} 32$, second semestre 2008, pp. 203-221}

\section{Introduction}

Dans cette communication, nous avons pour objectif de définir les contours théoriques $\mathrm{du}$ concept du don puis de les mettre à l'épreuve du champ humanitaire à travers la compréhension des principes qui guident la communication des Organisations Non Gouvernementales, en particulier celles de Handicap International.

Même si elle s'enrichit, à l'instar des travaux de Simondon, d'autres champs disciplinaires, notre approche se fonde essentiellement sur une perspective sociologique de l'objet d'étude. Le don, de notre point de vue, doit être appréhendé dans sa dimension institutionnelle, c'est-à-dire en tant que cadre social dont l'essence même est constituée par le caractère collectivement partagé des règles qui le régissent. Dès lors, il faudra d'abord nous intéresser à la nature des règles qui régissent le cadre du don et qui déterminent la manière dont les individus vont s'y engager. Celles-ci sont différentes de celles qui régissent la plupart des autres cadres sociaux (politique, sportif, médiatique etc.). En particulier, nous montrerons la place de l'émotion comme dimension constitutive du don voire comme condition nécessaire à sa réalisation et à celle de la communauté formée par les donateurs. Il nous faudra d'autre part interroger la place qu'entretient le don vis-à-vis du champ humanitaire. Nous verrons ainsi comment les ONG, en particulier Handicap International, s'emparent habilement, dans leurs communications télévisuelles, de ces modes de structuration de l'expérience pour amener progressivement le téléspectateur à se transformer en donateur.

\section{Presentation theorique de L'ObJet D'Etude : CADre Social ET DON}

\subsection{Cadres sociaux : principales caractéristiques théoriques}

Le don, de notre point de vue, doit être appréhendé en tant que cadre social (Goffman, 1991). Chez Goffman, l'organisation de la vie quotidienne s'agence autour de différents cadres sociaux lesquels structurent la vie de groupe et les relations des hommes entre eux. Ces cadres, que nous assimilons à travers notre expérience, nous fournissent en quelque sorte nos représentations et nos schémas d'interprétation du monde. Tout se passe comme si chacun d'entre nous disposait d'un stock de cadres et qu'il puisait spontanément dans ce répertoire de connaissances pour définir une situation et s'y engager. Cette remarque implique que nous avons intériorisé lors de notre existence un certain nombre de règles sociales qui sont partagées et reconnues par tous comme légitimes et que nous savons appliquer en fonction des cadres pertinents pour définir une situation : chacun implique des lieux, des façons de parler, 
des rôles parfaitement reconnaissables par autrui. Toute société, comme toute interaction, repose sur cet ordre et le propose comme allant de soi. Toute situation sociale requière donc un certain type de cadre qui dicte spontanément la manière dont nous devons régler notre attitude en son sein.

C'est donc en tant qu' " institution » (Ogien, 1999), qu'il faut concevoir la situation, c'est-à-dire en tant que modèle de référence, extérieur aux individus et dont ils vont se saisir pour évaluer dans le cours même de l'activité qui les réunit, la pertinence de leurs actes et de ceux d'autrui. Par exemple, nous savons nous comporter différemment selon que nous nous situons dans le cadre universitaire, le cadre sportif ou le cadre plus intime de l'univers amoureux. La vie sociale s'apparente ainsi à un répertoire de situations types pour lesquelles les participants à une interaction cherchent avant tout à endosser un rôle en accord avec la situation: nous n'attendons pas du professeur des universités qu'il fasse des déclarations publiques d'amour (cadre amoureux) à ses étudiants (cadre universitaire). Un tel comportement n'est pas toléré par le cadre universitaire dans lequel évolue le professeur qui doit, en l'occurrence, se contenter d'enseigner, transmettre des informations, évaluer etc.

Le maintien de l'ordre social (au sens de Durkheim) dépend de la réussite ou de l'échec de cet acte de représentation c'est-à-dire du bon respect, par les individus, des règles constitutives de ces cadres, lesquelles, si elles sont mises en faillite, mettent en péril les interactions sociales. Rien n'est donc plus sociologique que le concept de cadre qui intègre une nécessaire composante sociale et qui porte en lui une logique institutionnelle. Il en va de même pour le concept de don que nous voudrions ici définir.

\subsection{Le don : une institution fondée sur le lien social}

Depuis Marcel Mauss, il est communément admis que tout don implique un contre-don. Dans un essai réputé (1923), il désigne sous le nom de «potlatch » un type particulier de prestations sociales dans lesquelles s'affrontent, au cours de fêtes, les chefs de clan de sociétés tribales. Cette cérémonie marquée sous le sceau de la compétition consiste en dons ou destructions massives de richesses, chacun étant contraint de « rendre » ou détruire à son tour des quantités supérieures de richesses, sous peine de perdre son prestige et son pouvoir, et ceux de son clan : " Donner, c'est manifester sa supériorité, être plus, plus haut, magister ; accepter sans rendre ou sans rendre plus, c'est se subordonner, devenir client et serviteur, devenir petit, choisir plus bas (minister) » (Mauss, 1960, p. 269). Donner suppose donc un élément social qui contraint les individus qui reçoivent à un comportement réciproque dont le principe est magique, sinon sacré. L'échange, aussi paradoxal que cela puisse paraître, fait donc partie intégrante du don à tel point que le deuxième terme semble constituer un pléonasme du premier. Autrement dit, un don ne puise sa signification qu'à travers un second qui lui succède en retour.

Originellement, le don exige une contrepartie de même valeur, de façon à ce que le rapport social liant donneur et receveur ne soit pas compromis. Ce qui est en jeu dans les sociétés tribales décrites par Mauss, c'est bien le don en tant que source de pouvoir. Parce que recevoir sans donner en retour reviendrait à se constituer en partie faible ou dominée, à se 
déshonorer, bref, à perdre sa dignité. (Nous voyons ici combien la théorie des faces, chère à Goffman, aide à comprendre la logique du don présentée par Mauss). C'est dans la valeur du don en tant que lien social qu'il faut en appréhender la logique. Le véritable don n'est pas d'abord affaire de quantité. En fait, le don ne représente rien d'autre qu'un système qui transforme la nature des relations humaines. Donner, c'est avant tout créer, rituellement et symboliquement, un type particulier de relations à autrui : « [...] le don vise à la reproduction [...] sociologique, à l'établissement et au rétablissement du rapport social » (Caillé, 1994, p. 270). La symétrie de la relation n'est donc plus ce qui prime ; « le fait fondamental est que le lien importe plus que le bien » (Caillé, 1994, p. 236).

Toute relation de don implique donc la présence de trois éléments, un donataire, un donateur, et une chose donnée. Nous voudrions ici insister sur l'élément mental constitutif de cette relation (l'intention) qui permet notamment de distinguer l'acte de donation des autres actes de transferts physiques d'objets. En associant un donateur à un donataire par la médiation d'une chose donnée, la relation du don peut être assimilée à une sorte de modèle, d'archétype même de toutes les autres formes de relation triadiques (Descombes, 1996, p. 237). C'est donc sans conteste à Peirce qu'il convient de se référer pour évaluer la logique des relations qui fondent ce paradigme. La relation du don n'est pas seulement la relation qui associe deux acteurs sociaux suite au transfert d'un objet avec lequel ils auraient eu un quelconque rapport ; elle implique, en plus, un rapport d'intention entre la chose donnée et le donateur. Donner, c'est non seulement transmettre une chose à quelqu'un, mais c'est aussi avoir la conscience et l'intention d'attribuer à cette chose la valeur d'un don. Ignorer cette relation intentionnelle reviendrait à ignorer le fait triadique du don : « en laissant de côté l'intention, la description qui s'en tient aux faits bruts laisse de côté le don lui-même » (Descombes, 1996, p. 240). Omettre l'intention, c'est omettre le sens même de la relation ; c'est réduire l'acte de donation à une relation binaire ou dyadique résultant du simple transfert physique d'un objet d'une personne envers une autre. Ainsi, la relation du don implique de ne jamais séparer les liens de personne à personne et les liens de personne à chose qu'elle contient : « les personnes se lient par la médiation du don de l'objet, et l'objet est donné (pas seulement déplacé) par la médiation d'un lien des personnes »(Descombes, 1996, p. 245). Mauss parle quant à lui d'une espèce de mélange : «Tout va et vient comme s'il y avait échange constant d'une matière spirituelle comprenant choses et hommes [...] » (Mauss, 1960, p. 164).

L'explication de l'obligation de donner quand on possède, ou de rendre quand on a reçu, réside dans le sentiment conscient d'avoir cette obligation. Et l'origine de ce sentiment ne doit pas être recherchée dans des mécanismes psychologiques comme semble le croire Lévi Strauss, mais bien dans une logique institutionnelle qui préside au système du don. Autrement dit, si nous nous plaçons dans une perspective holiste au sein de laquelle le tout de l'échange est donné avant que ses parties soient présentes, c'est-à-dire avant même que les choses aient été présentées pour être données et rendues, c'est qu'il existe « une institution, un usage établi et transmis de génération en génération » (Descombes, 1996, p. 256). Le système du don est donc gouverné par une institution, un ensemble de règles déterminées que les gens estiment devoir être appliquées, de façon à la fois consciente et collective, pour diriger leur vie et 
maintenir le lien social qui les unit aux autres membres de leur société. C'est cette considération qu'il nous faut garder à l'esprit lorsque nous parlons du cadre du don. Ce dernier doit être appréhendé dans toute sa dimension institutionnelle, c'est-à-dire comme un cadre social dont l'essence même est constituée par le caractère normatif et collectivement partagé des règles qui le gouvernent.

\subsection{L'émotion comme dimension constitutive du don}

Comprendre comment opère le cadre du don nécessite de faire un détour par la philosophie de Simondon dont nous allons introduire les principaux traits définitoires qui intéressent notre discussion.

La thèse défendue par cet auteur s'inscrit en rupture avec les travaux entrepris par la psychanalyse. D'après lui, à la frontière entre conscience et inconscience, se situe la couche de la subconscience, siège de l'affectivité et de l'émotivité. L'action ou l'engagement d'un individu dans un groupe ou dans une société doit être compris en considérant l'état de cette couche de la subconscience : une étude sérieuse de l'individuation collective doit partir de l'analyse et de la reconnaissance de ces thèmes affectivo-émotifs, et non pas seulement d'une quelconque observation d'actions ou de représentations communément partagées (Simondon, 1989, p. 99).

En effet, l'organisation de la vie sociale semble avant tout reposer sur des considérations affectivo-émotives, lesquelles, lorsqu'elles font l'objet d'un consensus, cimentent la vie de groupe : «c'est au niveau des thèmes affectivo-émotifs, mixtes de représentation et d'action, que se constituent les groupements collectifs » (Simondon, 1989, p. 100). Tout collectif, c'est-à-dire toute agrégation de parole, se fonde sur cette communauté affective dont elle est le poumon. Et l'action des individus agit de fait en tant que manifestation concrète de ces échanges affectifs. Elle est ce par quoi l'affectivo-émotivité peut s'exorciser, c'est-à-dire s'accomplir et s'exprimer.

A la source de ce raisonnement, se trouve l'idée selon laquelle l'individu ne peut être pensé en dehors d'une double relation de dépendance à son psychisme et à son milieu. Et c'est aux fonctions affectivo-émotives qu'il faut attribuer le rôle fondamental de médiation entre ces deux univers, individuel et collectif. L'affectivité et l'émotivité assurent, en d'autres termes, une fonction connective entre la relation que l'individu entretient vis-à-vis de luimême et la relation qu'il entretient au monde : elles « seraient alors la forme transductive par excellence du psychisme, intermédiaire entre la conscience claire et la subconscience » (Simondon, 1989, p. 98).

Aussi, la charge de « réalité préindividuelle ", c'est-à-dire notre potentiel affectif, que nous portons tous de façon différenciée, lorsqu'elle s'individue comme être psychique, concourt à l'individuation collective, c'est-à-dire à l'intégration de l'individu en tant que membre d'un groupe : « l'individuation sous forme de collectif fait de l'individu un individu de groupe, associé au groupe par la réalité préindividuelle qu'il porte en lui et qui, réunie à celle d'autres individus, s'individue en unité collective » (Simondon, 1989, p. 19). 
Reste à savoir comment s'opère l'individuation du préindividuel chez les différents individus pour fonder le collectif : l'affectivité, quand elle se prolonge en émotion, devient une valeur collective à partir duquel un collectif peut être construit. En d'autres termes, l'émotion n'est ni plus ni moins que l'expression de l'affectivité dans le collectif. C'est alors l'activation des formes de l'affectivité, chez des sujets différents, qui permet d'attester l'existence du collectif, l'émotion permettant à l'individu d'entrer dans le collectif, de communiquer, c'est-à-dire de partager avec lui des valeurs communes; le collectif étant le médium incontournable à l'actualisation desdites émotions dans la mesure où elles impliquent obligatoirement la présence de l'individu à d'autres individus (Simondon, 1989, p. 107-116). Notre approche n'est pas très éloignée de celle des sociologues comme Mauss ou Durkheim, pour qui la notion d'obligation est centrale dans leurs travaux respectifs sur les émotions. Pour Mauss, l'expression des émotions est obligatoire dans certaines circonstances affectant le groupe d'appartenance. Dès lors, l'absence de manifestation publique de l'émotion est un affront à l'unité collective et doit être sanctionnée (Mauss, 1921, pp. 81 - 88). Dans son étude sur les rites de deuil, Durkheim (1968, p. 571) développe un point de vue similaire : la perte de l'un de ses membres requiert l'expression d'une émotion, laquelle constitue une contribution réglée à la solidarité du groupe en tant qu'elle témoigne de son degré d'attachement à l'entité collective. Etre attaché à la société dont nous faisons partie, c'est manifester ses émotions de manière conventionnelle sous peine de rompre les liens qui nous unissent à elle.

La prise en compte de ce point de vue est essentielle à la compréhension du cadre du don. Elle repose sur l'idée que l'émotion apparaît comme une dimension constitutive du don voire comme une condition nécessaire à sa réalisation et à celle de la communauté formée par les donateurs. Il s'agit ici de comprendre que l'engagement dans le cadre du don dépend d'un état affectivo-émotionnel spécifique et collectivement partagé.

\subsection{L'engagement dans le cadre du don}

Effectuer un don envers une association humanitaire, c'est donc permettre à nos émotions de se réaliser. L'acte de donner doit être considéré comme un acte par lequel l'émotion se manifeste dans la réalité transindividuelle. Il est le garant de sa concrétude. Autrement dit, émettre un chèque en faveur d'une organisation non gouvernementale, c'est beaucoup plus qu'effectuer un acte courant de la vie quotidienne : c'est aussi témoigner symboliquement de son état émotionnel, et, par voie de conséquence, de son appartenance à un collectif. Le don doit donc être entendu comme un instrument de la sédimentation collective. Il nous offre non seulement la possibilité d'extérioriser «matériellement » un état émotionnel, mais aussi de nous insérer dans un collectif, c'est-à-dire de nous engager aux côtés de tous ceux qui le composent. Le caractère parfois anonyme du don n'entrave pas la participation du donateur à ce collectif. Le collectif dont nous parlons ici n'implique aucunement le regroupement d'individus physiquement présents. Il s'identifie au contraire au Public que décrit Tardes et doit être entendu comme une «collectivité purement spirituelle » (1989, p. 13), comme un rapprochement, non pas de corps, mais d'émotions collectivement 
partagées. Il suffit au donateur anonyme de savoir que d'autres que lui ont partagé les mêmes émotions et effectué le même acte de donation pour qu'il se sache pris dans le collectif. Effectuer un don, anonyme ou pas, implique donc systématiquement la conscience, pour chacun des donateurs, d'appartenir à une communauté d'individus liés entre eux par la cohésion de leurs émotions ; et le don ne manifeste rien d'autre que notre détermination à prendre part à cette conscience collective.

En tant que cadre, le don est donc une forme d'organisation de l'expérience dont l'accès est déterminé, non pas par la position qu'occupe un individu dans l'espace social, mais par l'activation de certaines émotions à l'égard d'un discours ou d'une situation sociale à visée humanitaire. Les règles qui régissent l'entrée d'un individu dans le cadre du don se fondent alors sur une problématique légèrement différente de celles qui régissent l'ensemble des cadres sociaux. Il s'agit ici de comprendre que l'engagement dans le cadre du don n'est pas dépendant de nos styles de vie $^{1}$ (Esquenazi, 2002, p. 34), mais d'un état affectivoémotionnel spécifique et collectivement partagé. Donner, c'est assumer la concrétude d'une émotion ; c'est attester l'existence d'un état éprouvé. L'acte de donation renvoie donc à une modalité d'action particulière par laquelle un individu rend compte de ses émotions.

Le don n'est ni plus ni moins qu'un mode d'engagement pratique dans le cadre plus large de la solidarité humanitaire. Lorsque nous donnons, nous ne sommes plus observateurs d'une situation sociale, nous nous posons en acteurs de cette situation au même niveau que les autres donateurs, partenaires et membres de notre collectif. Participer aux émotions collectives permet ainsi aux individus d'entretenir les liens qui les unissent à la collectivité.

Le don, s'il ne peut être assimilé à une expression publique doit également être appréhendé comme une manifestation de l'engagement public des individus dans une entité collective, comme une contribution réglée à la solidarité d'un groupe. En d'autres termes, l'acte de donner révèle une conduite cadrée qui témoigne d'une posture significative dans un (micro) monde commun.

La notion de cadre implique, avons-nous dit, la présence d'acteurs sociaux qui entretiennent entre eux des relations spécifiques. Nous avons dit aussi qu'il est impossible de penser le cadre du don sans avoir la conscience qu'il se fonde sur un type de relation triadique : le don est une relation qui associe un donateur à un donataire grâce à une chose donnée (Descombes, 1996, p. 237). L'acte de donner en faveur de telle association plutôt que telle autre révèle ainsi l'existence d'une correspondance créée par la médiation de l'affectivité entre donateurs et ONG autour d'une cause commune. Par exemple, donner pour Handicap International, c'est d'une part témoigner de la propension de son affect à s'éprouver plus particulièrement pour la cause des enfants victimes d'amputation, mais c'est aussi

\footnotetext{
${ }^{1}$ Un cadre se situe au croisement du monde commun et de l'espace social nous dit J. P. Esquenazi. Or le monde commun est aussi espace social, hiérarchisé en styles de vie représentant des positions dans la structure ordonnée du social. Ces styles de vie qui déterminent un ensemble de «dispositions durables », constituent un habitus particulier (Bourdieu, 1980, p. 88). Celui-ci permet aux individus d'opérer une organisation spécifique de certains éléments de la réalité quotidienne. Ainsi, selon nos habitus, notre appréhension d'une même situation cadrée (une compétition sportive par exemple) pourra être différente. La structure des mêmes situations rencontrées par un professionnel du football ne sera pas perçue de la même manière par un profane.
} 
revendiquer ouvertement son appartenance et sa solidarité à l'égard de cette ONG. S'engager dans le cadre du don implique donc préalablement la conscience d'être engagé dans le cadre plus large de la solidarité. Nous allons voir ainsi comment les ONG, en particulier Handicap International, s'empare habilement, dans ses communications télévisuelles, de ces modes de structuration de l'expérience pour amener progressivement le téléspectateur à se transformer en donateur. Cette opération s'opère par glissements successifs de cadres sociaux et s'articule autour de l'activation des émotions.

\section{APPROChe anAlytiQue Des CAMPAgnes PUblicitaires et MODElisation CONCEPTUELLE}

\subsection{Cadre du don et publicité}

Traditionnellement, le cadre publicitaire obéit à deux logiques fondamentalement différentes et cependant complémentaires. La première est liée au statut économique du film publicitaire ; la seconde à son statut culturel. Cette approche s'appuie sur les recherches entreprises par Fiske à l'égard de la télévision. Cet auteur montre que les textes filmiques, en fonction de leur nature, doivent être envisagés autour de l'opposition entre logique économique (commerciale) et culturelle (artistique).

Il apparaît clairement que les films publicitaires pour les associations humanitaires ne peuvent se situer dans l'un ou l'autre cadre, et cela pour deux raisons : d'une part parce que Handicap International n'a rien à vendre : la question humanitaire ne peut faire l'objet d'une approche en terme de produits ; d'autre part parce que son entreprise est trop sérieuse pour que ses films soient repris à l'intérieur d'une économie culturelle de la publicité. De ce point de vue, les films qui portent sur l'objet humanitaire sont beaucoup plus proches des films institutionnels où la publicité cherche, non pas à faire valoir directement les produits, mais les entreprises qui les inventent, qui les fabriquent ou qui les distribuent (Brochand \& Landrévie, 1989, p. 76). Bien qu'elle soit institutionnelle, la publicité humanitaire est avant tout Publicité. Si les films pour venir en aide aux enfants afghans viennent s'intercaler entre d'autres spots de nature complètement différente, c'est sans doute parce que, d'un point de vue formel, ils ne s'en différencient guère. En termes goffmaniens, nous dirons simplement que la publicité humanitaire n'est rien d'autre qu'une manipulation (ou modalisation) du cadre du don sur le cadre publicitaire traditionnel : les films publicitaires que nous voyons couramment à la télévision servent en effet de modèle, ou de fondement, pour tous les films qui font appel à la générosité des téléspectateurs.

\subsection{Ritualisation de la mise en scène publicitaire}

Nous voudrions ici proposer un modèle qui permette d'appréhender de façon synthétique comment s'opère la mise en scène des campagnes étudiées. Le travail de Handicap International n'est pas tellement éloigné de la tâche d'une entreprise qui doit vendre son produit afin de se différencier de ses concurrents dans un champ spécifique. La principale 
mission à laquelle se voue l'ONG en question est à la fois de lutter contre les mines antipersonnel et d'aider les victimes de ces armes à retrouver leur dignité en leur offrant des prothèses et en leur réapprenant à marcher. Mines et enfance constituent ainsi les deux éléments cristallisants de l'identité discursive « Handicap International » qui nous permettent à la fois de la reconnaître et de la différencier d'autres institutions à vocation humanitaire comme La Croix-Rouge ou Vétérinaires Sans Frontières.

Le schéma qui suit a pour fonction de mettre en évidence la ritualisation de ces situations de communication qui fondent l'institutionnalité de Handicap International ${ }^{2}$.

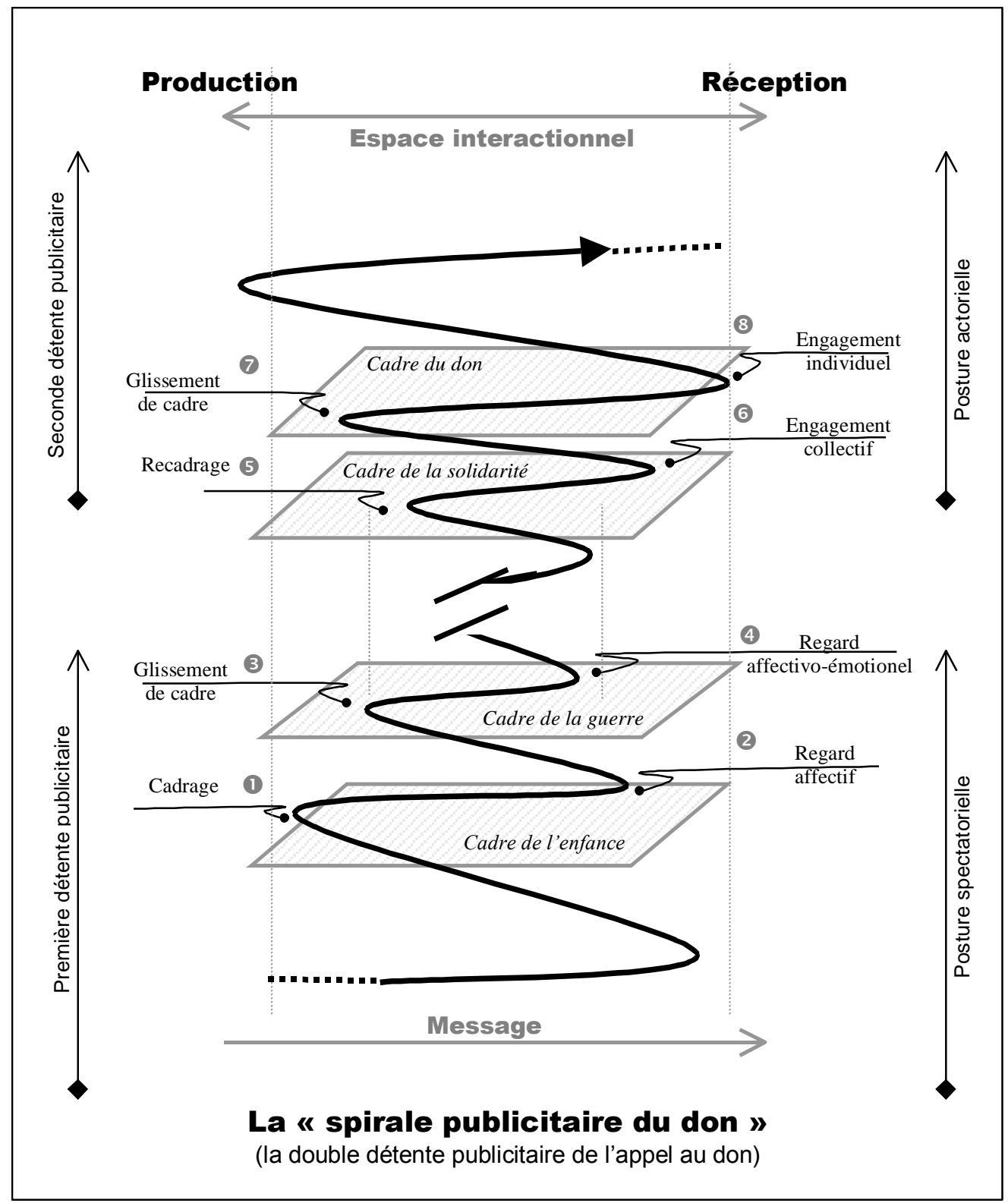

\footnotetext{
${ }^{2}$ L'étude porte sur un corpus de 8 campagnes télévisuelles comprises entre 1992 et 2002 que nous ne présentons pas ici en intégralité. Pour l'analyse complète voir C. Dessinges, 2002.
} 
La spirale symbolise l'activité de lecture du téléspectateur. Sa forme originale traduit le processus actif et non univoque de réception; elle matérialise tout à la fois l'attention portée aux films publicitaires et l'émotion que ces derniers suscitent. Elle représente la dynamique qui va porter le téléspectateur à s'intégrer dans le cadre du don, c'est-à-dire à se transformer en donateur. La spirale du don s'inscrit dans un schéma complexe de la communication, où l'on assiste à un va-et-vient entre les préoccupations et les émotions du producteur et celles-là même qu'il entend faire progressivement partager au récepteur- téléspectateur par différents processus de cadrage. La dynamique publicitaire de l'appel au don s'articule en deux temps : c'est pour cela que l'on peut parler de «double détente publicitaire de l'appel au don » (échelle de gauche sur le graphe). De façon surprenante, ces deux étapes font également référence aux deux grandes orientations de l'activité des ONG humanitaires : l'appel à réaction (première étape) et l'appel à l'action ou au don (seconde étape). Cette notion rend compte du dédoublement postural dont le téléspectateur est l'objet.

La première phase (première détente publicitaire) consiste à maintenir l'attention affective de l'instance réceptrice : on lui propose ici une simple posture spectatorielle. La seconde étape (deuxième détente publicitaire) vise quant à elle à le faire passer du stade d'observateur à celui d'acteur - engagé (donateur), via l'émotion et la pitié éprouvées. Le passage à la posture actorielle sera opéré habilement par glissements successifs de cadres.

$\mathrm{Au}$ centre du schéma, la superposition des cadres rend compte de leur apparition sur l'échelle temporelle : on se situe ici au niveau de l'objet publicitaire.

L'échelle de droite correspond aux principaux modes d'engagement du téléspectateur aux différents moments des films : au niveau affectif (2), on requiert d'abord de lui qu'il s'implique affectivement à la fois par la connaissance et la conscience (1) d'un premier cadre de référence qui le sous-tend : celui de l'enfance ; le second regard, dit affectivo-émotionnel (4), rend compte de l'activation d'un certain état émotionnel engendré par la superposition (3) de deux cadres « ennemis », celui de la guerre et celui de l'enfance. A ce niveau de lecture du film publicitaire, le téléspectateur est amené à modifier radicalement sa posture, ce qui est matérialisé, au centre du schéma, par la rupture de spirale, qui est aussi celle de son expérience cognitive.

Cette rupture légitime par ailleurs la mise en place (5) du cadre de la solidarité positionnant le téléspectateur en tant que partenaire d'un collectif (6). Enfin le passage du cadre de la solidarité à celui du don (7) lui propose d'assumer un rôle spécifique dans ce collectif : celui de donateur(8).

La double détente s'effectue dans un laps de temps très court : celui du déroulement de la publicité. Plus l'intensité de la transition entre les deux «détentes » est forte, plus «l'effet ressort» est important et donc l'appel au don susceptible d'être suivi par un grand nombre de téléspectateurs. Tout l'intérêt pour les concepteurs des spots publicitaires est donc de gérer les différents glissements de cadres qui aiguiseront l'attention et l'émotion du téléspectateur.

Si la spirale du don semble parfaitement compatible avec toute sorte de communication humanitaire, la première détente qui joue sur le glissement du cadre de l'enfance (pureté) à celui de la guerre (horreur) est la caractéristique exclusive des campagnes de Handicap International ; et c'est bien normal car la problématique des mines antipersonnel est le cheval de bataille de l'ONG. 
Nous voudrions maintenant expliciter davantage le fonctionnement de ce modèle, en prenant soin, à chaque fois, de considérer chacun des éléments qui figurent dans le modèle. Dans cette entreprise, nous choisirons les différents cadres sociaux comme points d'ancrage de notre réflexion.

\subsubsection{L'émotion comme résultant de la cohabitation de cadres ennemis}

Comme pour toute identification d'événements, l'interprétation d'une séquence

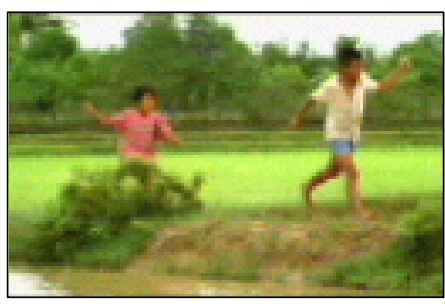
télévisuelle nécessite un travail d'identification de ce qui s'y passe. La première phase dont il est question dans la construction de chacun des films publicitaires est la détermination d'un cadre particulier. Dans l'ensemble des publicités étudiées, l'agencement des divers éléments sonores (musique enfantine, énoncés comme : «rire, chanter, jouer etc ») et visuels (des enfants qui jouent, cerf volants etc) manifeste ainsi des jeux de langage (Wittgenstein, 1961) qui inscrivent notre expérience de réception filmique dans le cadre de l'enfance. La mise en scène discursive ainsi mobilisée induit un mode de communication spécifique, dans lequel les acteurs sociaux sont supposés avoir une connaissance partagée des valeurs positives liées à l'affectif et à l'innocence.

Le premier regard affectueux que le téléspectateur est conduit à éprouver en début de campagne va progressivement se transformer avec l'entrée en scène d'éléments appartenant

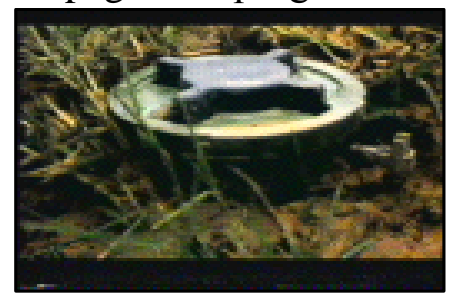
au registre de la guerre (présence de mines anti personnel sur le terrain de jeu des enfants, métonymie de l'explosion etc). Dans la mesure où nous sommes dépositaires d'un savoir relatif à la qualité minée du terrain, il nous est impossible de poursuivre notre activité de lecture avec le même regard affectif. Les effets perlocutoires engendrés par le fait de voir la mine ont en effet suscité de notre part un sentiment d'effroi, et c'est avec une implication nouvellement définie et teintée d'inquiétude que nous continuons à regarder les enfants courir. L'entrée en scène de l'arme mutilante a en effet pour objectif de modifier sensiblement le cadre de l'enfance initialement appliqué à la lecture du film. Ce dernier est désormais coloré d'une connotation peu singulière : dans certaines régions du monde, les enfants évoluent sur des terrains minés qui mettent leur vie en danger. En d'autres termes, et dans le type de pays qui est ici présenté (Asie), les mines sont des éléments du cadre de la guerre qui semblent cohabiter avec le cadre de l'enfance.

Ces références, dans l'image, au cadre de la guerre ne peuvent que surprendre le lecteur

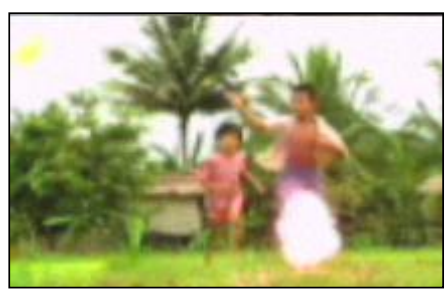
qui est convié à assister à la scène de l'explosion. Dans les différentes campagnes, l'apparition de l'explosion agit d'une part comme rupture cognitive et requiert d'autre part inéluctablement 
l'expression de sentiments proches de la pitié : l'association du cadre de la guerre avec le cadre de l'enfance constitue à cet égard le moment fondateur de l'émotion télévisuelle.

L'émotion éprouvée à la lecture des campagnes institutionnelles nécessite alors un recadrage de l'activité en cours : le téléspectateur est dans l'expectative de nouvelles informations susceptibles de l'aider à donner du sens à son expérience télévisuelle. Dans ce contexte d'interaction médiatisée, c'est le discours de la voix-off qui va permettre au téléspectateur de formuler une interprétation correcte de la situation.

Chaque explosion déclenche ainsi l'apparition de l'instance énonciatrice. En même tant qu'apparaît à l'écran le logo d'Handicap International, plusieurs énonciations vont être accomplies par un locuteur : elles ont en commun d'expliciter le sens de l'action en déclarant, sous forme de constat, un état de choses : «Handicap International réapprend aux enfants à marcher » ou «Dans plus de 60 pays, les mines tuent et mutilent même en temps de paix » ou encore «Toutes les vingt minutes, un paysan, une femme ou un enfant, est fauché par une mine ». Le locuteur est ici un être universel dont le rôle est d'abord de constater un état de fait dont la véracité est attestée par des références précises : les mots « toutes les vingt minutes » conférant par exemple à la situation une authenticité toute mesurée.

\subsubsection{De la solidarité au don}

Dans la mesure où les informations énoncées par le locuteur des films demeurent intolérables à plus d'un titre, elles légitiment la mise en place du cadre de la solidarité. Celuici a quelque chose de fortement relationnel. D'abord, parce qu'il a pour fonction d'interpeller directement le téléspectateur, ensuite parce qu'il implique une orientation nouvelle dans les positions que chacun des acteurs de l'interaction télévisuelle doivent adopter les uns vis-à-vis des autres.

Le cadre de la solidarité, en succédant à l'émotion, prend place à travers différents moments énonciatifs. Des énoncés comme «Arrêtons de jouer avec la vie des enfants » ou «Avec Handicap International, aidons les hommes à reprendre la marche de leur vie » attribuent explicitement aux individus concernés par la forme pronominale du verbe une action collective. En exprimant un énoncé dont le caractère est universel, c'est-à-dire qui a la qualité de pouvoir être accompli par chacun d'entre nous car ayant la propriété de se présenter comme indiscutable, cette phrase s'inscrit dans le cadre de la solidarité; ceci implique qu'elle propose à chacun d'entre nous une action identique : plus exactement elle nous associe dans une action collective dont la finalité nous est commune, comme si nous formions une seule entité.

La forme pronominale de la première personne du pluriel engage dans une activité solidaire l'énonciateur et les membres de son auditoire aux côtés de l'association Handicap International qui figure dans l'énonciation comme le poumon de l'opération d'assistance.

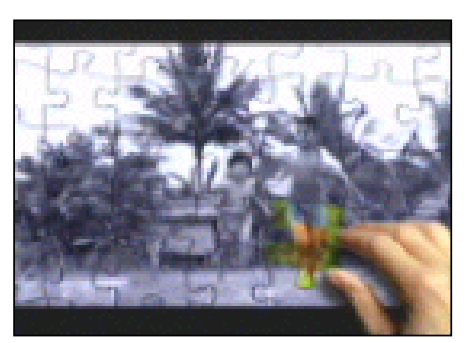
L'adverbe « avec » renforce cette idée de nécessité d'agir collectivement. Il est assez facile de décrire dans l'image ellemême des éléments qui manifestent ce cadre, la main étant l'élément principal. La définition de ce nouveau cadre de 
l'entraide et de la solidarité détermine ainsi un déplacement énonciatif dans lequel responsable énonciatif et interlocuteur tendent à fondre en une seule entité : comme si l'acte de la main était accompli aussi bien par le responsable de l'énonciation que par le téléspectateur. Dans cette relation en effet, chacun doit être pris sous la description d'une partie dans un tout ; chaque partie étant le maillon d'une chaîne qu'Handicap International se plaît à relier.

Les actes d'interpellation accomplis dans le cadre de la solidarité ont ainsi pour effet de mobiliser la portée universelle de notre citoyenneté : ils procèdent d'une interpellation éthique, laquelle « enveloppe un appel à l'universalité concrète que tout être humain porte en lui » (Resweber, 1998, p. 237). C'est donc en tant que citoyen du monde qu'il faut nous positionner. Dans cette perspective, la performance accomplie par la publicité est de convertir la posture du téléspectateur en un membre de la communauté du monde considéré du point de vue de ses obligations morales et de ses devoirs civils. Dès lors, il se trouve engagé dans une forme de relation qui s'apparenterait à la solidarité mécanique (Durkheim, 1978) définie à la fois par la cohésion des émotions, et l'adhésion unanime à certaines valeurs éthiques propres à l'humanité.

Est alors offerte au téléspectateur une position idéale dans laquelle il peut déployer toute son humanité aux côtés de ceux qui lui ressemblent. Et dès lors qu'il assume cette posture, il se trouve obligé, dirait Mauss (1960, p. 159). D'où le recours au cadre du don et aux actes d'appel à mobilisation qui le fondent.

Chacune des dernières séquences de films met en place le cadre du don. Les dernières

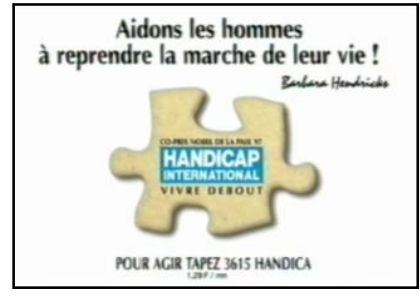
énonciations orales ou retranscrites à l'écran, en ayant recours de manière plus ou moins explicite aux formes pronominales de la deuxième personne du pluriel («Pour dire Non, 3615 Handica »; «Pour agir, tapez 3615 Handica ») témoignent de la détermination des publicités à faire penser au téléspectateur qu'il est au centre de son dispositif. Il devient l'interlocuteur principalement visé. On requiert de lui, sur un ton injonctif, qu'il manifeste concrètement son engagement par la médiation d'un don en faveur de Handicap International. Ce type d'énoncé redéfinit les rôles que chacun des interlocuteurs doit avoir dans le cadre de la solidarité : locuteur, Handicap International et spectateurs sont ainsi figurés de façon distincte dans l'énonciation finale. La présence implicite de la forme pronominale de la deuxième personne du pluriel inscrit en effet le spectateur au centre du dispositif de mobilisation et dégage le locuteur d'une responsabilité sociale qu'il a déjà prise par le fait même de parler. Enfin, l'organisation humanitaire Handicap International figure dans l'énonciation comme le médiateur incontournable sans lequel nous ne pouvons agir. L'inscription du logo témoigne aussi du rôle central joué par l'association dans cette opération. La mise en place des énonciations finales propose donc une nouvelle posture au téléspectateur dans laquelle il est confronté à sa propre conscience : la responsabilité d'agir ne concerne plus seulement celui qui parle, mais aussi celui qui regarde. On lui insigne ainsi une qualité nouvelle : celle de téléspectateur responsable. Assumer cet état, c'est se transformer en donateur. 


\section{Conclusion}

A l'intérieur du champ humanitaire, il apparaît que le concept de don fasse l'objet d'une investigation particulière. En tant que cadre social, le système du don, en possède les conditions d'existence universelles : il est gouverné par une institution, un ensemble de règles que les individus ont intériorisées et qui leur permettent par exemple de différencier l'acte de donation de l'acte d'achat, notamment grâce à la reconnaissance d'un rapport d'intention entre la chose donnée et le donateur. Cependant, le don constitue un cadre original dont les propriétés spécifiques le distinguent de ses principaux voisins : ses conditions de réalisation ne dépendent pas d'une posture significative dans l'espace social mais d'un état affectivoémotionnel spécifique et collectivement partagé. C'est donc par une opération de transformation de l'affectivité en émotion que s'opère le processus d'individuation collective par lequel peuvent émerger des communautés affectives.

Les ONG l'ont bien compris. Si l'acte de donation est un acte individuel, ses conditions de réalisation dépendent en amont de l'existence d'une agrégation de parole c'est-à-dire d'un collectif. Il en résulte que l'étude du cadre du don est indissociable de l'analyse du cadre de la solidarité qui le précède. Dans ce contexte, il est singulier de rencontrer, dans le champ humanitaire, des campagnes télévisuelles qui jouent avec les cadres sociaux de notre monde commun pour générer l'émotion des téléspectateurs, véritable déclencheur de ce cadre de la solidarité. A cet égard, le recours au cadre de l'enfance est assez symptomatique de cette volonté. Il est vrai qu'il constitue par essence le siège de l'affectivité. Pour le transformer en émotion, une recette universelle consiste à l'associer à un cadre aux propriétés opposées, un cadre ennemi comme celui de la guerre. Nous pouvons en imaginer d'autres...

\section{Bibliographie}

Caillé, A., Don, intérêt et désintéressement : Bourdieu, Mauss, Platon et quelques autres, La découverte/Mauss, Paris, 1994, 304 p.

Descombes, V., Les institutions du sens, Les éditions de Editions de Minuit, Paris, 1996, 349 p.

Durkheim, E., Les formes élémentaires de la vie religieuse, PUF, Paris, 1968 (1912), $64 \mathrm{p}$.

Durkheim, E., De la division de travail social, PUF, Paris, 1978 (1893), 416 p. 
Esquenazi, J.P., L'écriture de l'actualité, PUG, 2002, Grenoble, 184 p.

Esquenazi, J.P., \& Walter, J., «Pouvoirs et quête de légitimité », in Le Téléthon, Scène Intérêts - Ethique, Editions l'harmattan, Paris, 1998, pp. 131-187.

Goffman, E., Les cadres de l'expérience, Editions de Minuit, Paris, 1991(1974), 573 p.

Mauss, M., « L'expression obligatoire des sentiments », in Essais de sociologie, Editions de Minuit, Paris, 1968 (1921), pp. 81-88.

Mauss, M., «Essai sur le don » in Sociologie et anthropologie, PUF, 1960 (1923), Paris, pp. 145-279.

Resweber, J. P., «L'éthique du téléthon », in Le Téléthon : Scène - Intérêts - Ethique, Editions l'harmattan, Paris, 1998, pp. 227-246.

Simondon, G., L'individuation psychique et collective, Aubier, Breteuil-sur-Iton, 1989, $293 \mathrm{p}$.

Wittgenstein, L., Tractatus logico-philosophicus, (suivi de) Investigations philosophiques, Gallimard, Paris, 1961, 324 p. 